$\frac{47 / .94859}{46^{-9}}$ UNIVERSITY OF WISCONSIN

Center for Plasma Theory and Computation REPORT

\title{
Anomalous Transport Equations in Toroidal Plasmas
}

\section{A.I. Smolyakov' I.D. Callen and A. Hiroset}

Nuclear Engineering \& Engineering Physics and I'hysics Departments

University of Wisconsin-Madison

Madison, Wisconsin 53706 USA

'University of Saskatchewan,

Saskatoon, Saskatchewan S7N OWO CANADA.

\section{March 1994}

UW-CPTC 94-2

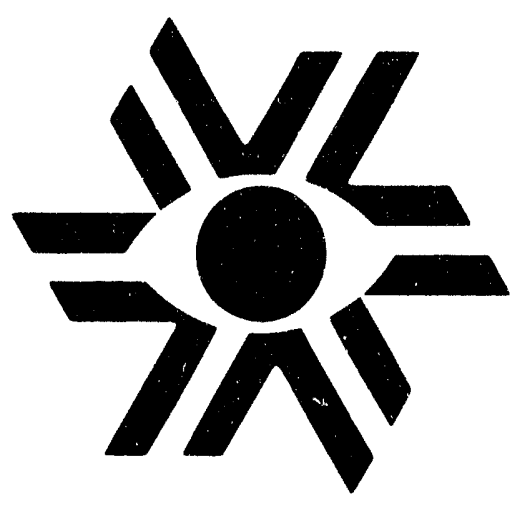

Madison, Wisconsin 53706-1687 
This report has been reproduced directly from the best available copy.

Available to DOE and DOE contractors from the Office of Scientific and Technical Information, P.O. Box 62, Oak Ridge, TN 37831; prices available from (615) 576-8401, FTS 626-8401.

Available to the pt:blic from the National Technical Information Service, U.S. Department of Commerce, 5285 Port Royal Rd., Springfield, VA 22161.

This report was prepared as an account of work sponsored by an agency of the United States Government. Neither the United States Government nor any agency thereof, nor any of their employees, makes any warranty, express or implied, or assumes any legal liability or responsibility for the accuracy, completeness, or usefulness of any information, apparatus, product, or process disclosed, or represents that its use would not infringe privately owned rights. Reference herein to any specific commercial product, process, or service by tradename, trademark, manufacturer, or otherwise, does not necessarily constitute or imply its endorsement, recommendation, or favoring by the United States Government or any agency thereof. The views and opinions of authors expressed herein do not necessarily state or reflect those of the United States Government or any agency thereof. 


\title{
ANOMALOUS TRANSPORT EQUATIONS IN TOROIDAL PLASMAS
}

\author{
A.I. Smolyakov ${ }^{\dagger}$, J.D. Callen and A. Hirose ${ }^{\dagger}$ \\ University of Wisconsin, Madison. IVI 53706-1687, USA \\ $\dagger$ University of Saskatchewan, Saskatoon, STN 0W0, Canada
}

November 9, 1993

\begin{abstract}
Reduced transport equations for a toroidal plasma with fluctuations are derived. These equations include the effects of both anomalous and standard neoclassical transport, and allow clarification of the structure of convective fluxes caused by electrostatic and magnetic fluctuations. Special attention is paid to the combined effects of fluctuations and toroidicity on the transport. The formulation retains the effects of a magnetic field inhomogeneity on the anomalons transport. It is shown that phase space diffusion cansed hy the gradient. in the equilibrium magnetic field appears as a pinch flux in the real space.
\end{abstract}

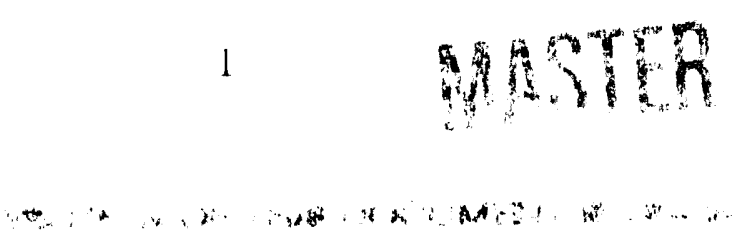




\section{Introduction}

Numerous transport analyses carried out for tokamaks have indicated the existence of energy [1] and/or density [2] pinches. In order to model experimental data, it is often necessary to assume that the total flux of quantity $X$ ( $X=$ temperature or density) consiscs of diffusive and convective parts, $\boldsymbol{\Gamma}_{X}=-D \nabla X-\mathbf{V}_{0} X$, where $\mathbf{V}_{0}$ is the pinch velocity. The neoclassical Ware-Galeev pinch is in general too small to explain the pinch velocities inferred in experiments, and various mechanisms have been proposed in terms of turbulence-induced anomalous pinches. In slab geometry, the pinch effect may be attributed either to wave convection [3], or to off-diagonal cross terms in the matrix of transport coefficients [4]. In more realistic toroidal geometry, pinch effects have been found in anomalous transport due to toroidal electrostatic modes [5]. However, a general formulation for anomalous transport equations in toroidal plasmas, including magnetic as well as electrostatic fluctuations, is yet to be made. This study is an attempt to formulate transport equations which can be used as an initial basis for investigation of anomalous transport in a toroidal system and include the effects of both toroidicity and fluctuations. (Naturally such equations should include also neoclassical transport as a limiting case.) In the present communication, we investigate the anomalous transport equations in full toroidal geometry with an emphasis on some new terms due to the combined effects of fluctuations and toroidicity. These new effects reveal themselves as pinch terms in the heat and density fluxes. Using a drift-kinetic equation it is shown that this pinch effect is associated with the diffusion in the phase space caused by an inhomogeneity in the equilibrium magnetic field.

For a Hamiltonian system, the Fokker-Planck equation for the evolution of a mean distribution function is purely diffusive withont friction terms [6]. In the present investigation, the electromagnetic fields are prescribed, and the effects of wave emission, which leads to a non-zero dynanic friction force [7], will be ignored. Considering the diffusivity matrix in phase siace, it will be shown that in addition to the standard terms proportional to the gradients of plasma temperature and density, the phase space fluxes contain contributions from the gradient of the magnetic field. When the phase space flux is projected onto configuration space by taking the appropriate moments of the drift kinetic equation, the diffusion caused by a magnetic nonuniformity appears as pinch effects in the real space. In order of magnitude, the 
pinch flux scales as $L_{n} / L_{B}$ with respect to the diffusive Hux, where $L_{n}$ is the characteristic length scale of plasma nonuniformity, and $L_{B}$ is that of the magnetic field. It is noted that the phase space volume conservation is important to secure the Hamiltonian nature of the averaged equations of the particle motion. We discuss the different forms of the drift-kinetic equation paying particular attention to this property.

\section{Drift-Kinetic Equation}

The general form of drift-kinetic equation can be written as [ 8$]$

$$
\frac{\partial f}{\partial t}+\nabla \cdot\left(\frac{d \mathbf{R}}{d t} f\right)+\frac{\partial}{\partial v_{\|}}\left(\frac{d v_{\|}}{d t} f\right)+\frac{\partial}{\partial v_{\perp}^{2}}\left(\frac{d v_{\perp}^{2}}{d t} f\right)=0,
$$

where $\mathbf{R}$ is the guiding center coordinate. We start with the guiding center equations written in the reference frame moving with the fluid velocity [9], $\mathbf{V}=\int \mathbf{v} f(\mathbf{r}, t, \mathbf{v}) d \mathbf{v}, \mathbf{v}=\mathbf{v}^{\prime}+\mathbf{V}$, where $\mathbf{v}^{\prime}$ is the random particle velocity in the moving frame. (The superscripts will be dropped below, so that all $v, v_{\perp}$ and $v_{\|}$are random velocities.) Averaged equations can be obtained by applying the standard procedure [10] to the original equation of particle motion. In the low $\beta$ limit $(\nabla \times \mathbf{B} \simeq 0)$, they are given by

$$
\begin{gathered}
\frac{d}{d t} \mathbf{r}=v_{\|} \mathbf{h}+\frac{1}{\omega_{B}} \mathbf{F} \times \mathbf{h}+\frac{1}{\omega_{B}}\left(\frac{v_{\perp}^{2}}{2}+v_{\|}^{2}\right) \mathbf{h} \times \Gamma \ln B . \\
\frac{d}{d t} v_{\|}=\mathbf{F} \cdot \mathbf{h}+\frac{v_{\perp}^{2}}{2} \nabla \cdot \mathbf{h}+\frac{v_{\|}}{\omega_{B}} \mathbf{F} \cdot \mathbf{h} \times \nabla \ln B-v_{\|} \mathbf{h} \cdot \mathbf{h} \cdot \nabla \mathbf{V}, \\
\frac{d}{d t} v_{\perp}^{2}=-v_{\perp}^{2} v_{\|} \nabla \cdot \mathbf{h}+\frac{v_{\perp}^{2}}{\omega_{B}} \mathbf{h} \cdot \nabla \times \mathbf{F}+\frac{v_{\perp}^{2}}{\omega_{B}} \mathbf{F} \cdot \mathbf{h} \times \nabla \ln B \\
-v_{\perp}^{2}(\nabla \cdot \mathbf{V}-\mathbf{h} \cdot \mathbf{h} \cdot \nabla \mathbf{V}) .
\end{gathered}
$$

Here $\omega_{B}=q B / m c$ is the cyclotron frequency. $r$ is the position of the guiding center in the moving frame and $h$ is the mit vector along the magnetic field. Terms of the order $1 / \omega_{B}^{2}$ and higher (including inertial drift) have been neglected here because they are unimportant for the calculations of the 
anomalous fluxes. It can be readily checked that Eqs. (2)-(4) conserve the phase space volume in the form

$$
\nabla \cdot\left(\frac{d \mathbf{r}}{d t}+\mathbf{V}\right)+\frac{\partial}{\partial v_{\|}}\left(\frac{d v_{\|}}{d t}\right)+\frac{\partial}{\partial v_{\perp}^{2}}\left(\frac{d v_{\perp}^{2}}{d t}\right)=0
$$

The function $\mathbf{F}$ can be considered as an effective electric field in the moving frame and is given by

$$
\mathbf{F}=\frac{\nabla p}{m n}+\frac{\nabla \cdot \boldsymbol{\Pi}}{m n}
$$

Eqs.(2)-(4) can be rewritten in terms of the kinetic energy, $\mathcal{E}=i^{2} / 2$, and the magnetic moment, $\mu=v_{\perp}^{2} / 2 B$,

$$
\begin{aligned}
\frac{d}{d t} \frac{v^{2}}{2}=v_{\|} \mathbf{F} \cdot \mathbf{h} & +\frac{v_{\perp}^{2} / 2+v_{\| 1}^{2}}{\omega_{B}} \mathbf{F} \cdot \mathbf{h} \times \nabla \ln B-\frac{v_{\perp}^{2}}{2 \omega_{B}} \mathbf{h} \cdot \nabla \times \mathbf{F} \\
& -\frac{v_{\perp}^{2}}{2} \nabla \cdot \mathbf{V}-\left(v_{\|}^{2}-\frac{v_{\perp}^{2}}{2}\right) \mathbf{h} \cdot \mathbf{h} \cdot \nabla \mathbf{V}, \\
\frac{d}{d t} \frac{v_{\perp}^{2}}{2 B} & =-\frac{v_{\perp}^{2}}{2 B \omega_{B}} \mathbf{h} \cdot \nabla \times \mathbf{F}-\frac{v_{\perp}^{2}}{2 B} \mathbf{V} \cdot \nabla \ln _{11} B \\
& -\frac{v_{\perp}^{2}}{2 B}(\nabla \cdot \mathbf{V}-\mathbf{h} \cdot \mathbf{h} \cdot \nabla \mathbf{V}) .
\end{aligned}
$$

We have used in Eqs.(7)-(8) the fact that in the low i approximation the strength of magnetic field is constant, $\partial B / \partial t=0$. Eqs. $(\tau)-(\delta)$ are simplified versions of those derived by Hazeltine and Ware [9]. Simplification is in ignoring the effects of the polarization drift (which is of the order of $V_{E} \omega / \omega_{B}$ ) and in using the low $\beta$ approximation (so that $(\mathrm{h} \cdot \Gamma) \mathrm{h} \simeq \Gamma \ln B$ ). A major difference is the presence of the term $-v_{\perp}^{2} \mathbf{h} \cdot \nabla \times \mathbf{F} / 2 B \omega_{B}$ on the right-handside of our Eq. (8). This term is important to secure the adiabatic moment conservation when the fluid velocity $\mathbf{V}$, in addition to the $\mathbf{E} \times \mathbf{B}$ drift, has the diamagnetic and viscosity components:

$$
\mathbf{V}=V_{\|} \mathbf{h}+\mathbf{V}_{E}+\mathbf{V}_{p}+\mathbf{V}_{\pi}
$$

where

$$
\mathbf{V}_{E}=\frac{c}{B} \mathbf{E} \times \mathbf{h}, \quad \mathbf{V}_{p}=\frac{c}{\epsilon n B} \mathbf{h} \times \nabla p, \quad \mathbf{V}_{\pi}=\frac{1}{\epsilon n B} \mathbf{h} \times \nabla \cdot \boldsymbol{\Pi} .
$$


The viscosity is approximated by its parallel component (the contribution of the gyroviscosity is of the order of the polarization drift and is neglected here),

$$
\Pi_{\|}=\pi_{\|}\left(\mathbf{b} \mathbf{b}-\frac{1}{3} \mathbf{I}\right)
$$

Note that in [9] the approximation $\mathbf{V}_{\perp}=\mathbf{V}_{E}$ has been used. In this approximation the first term on the r.h.s. of Eq. (8) identically vanishes. It can be shown that the adiabatic moment in $\mathrm{Eq} .(8)$ is conserved if $\mathrm{Eq}$. (6) for $\mathbf{F}$ and Eq. (9) for $\mathbf{V}$ are used.

For our purpose, it is more convenient to rewrite Eqs. (2), (7) and (8) by means of Eqs. (6) and (9). Then we obtain

$$
\begin{aligned}
& \frac{d \mathbf{R}}{d t}=v_{\|} \mathbf{h}+\mathbf{V}_{E}+\mathbf{V}_{d}, \\
& \frac{d v_{\|}}{d t}=\frac{q}{m} \mathbf{E} \cdot \mathbf{h}+\frac{1}{2} v_{\perp}^{2} \nabla \cdot \mathbf{h}+v_{\|} \mathbf{V}_{E} \cdot \nabla \ln B . \\
& \frac{d v_{\perp}^{2}}{d t}=-v_{\perp}^{2} v_{\|} \nabla \cdot \mathbf{h}+v_{\perp}^{2} \mathbf{V}_{E} \cdot \nabla \ln B .
\end{aligned}
$$

Here $\mathbf{V}_{d}=\frac{v_{\perp}^{2} / 2+v_{\|}^{2}}{w_{B}} \mathbf{h} \times \nabla \ln B$ is the magnetic drift velocity. Eqs. (11)(13) can be also obtaired by directly reducing the more complete guiding center equations by Sivukhin [8] to the lowest order in the small parameter $\beta=8 \pi p / B^{2} \ll 1$. The drift-kinetic equation (1) with Eqs. (10)-(12) has been directly derived in [11] and also in [12].

In an inhomogeneous magnetic field the divergence of the $\mathbf{E} \times \mathbf{B}$ drift is not zero, $\nabla \cdot \mathbf{V}_{E} \simeq-2 \mathbf{V}_{E} \cdot \nabla \ln B$. The last terms in Eqs. (11)-(12) are important to cancel this term in the phase space volume conservation the magnetic drift velocity is divergence-free in the low ; approximation). These terms in Eqs. (11)-(12) are responsible for the diffusion in velocity space [13]. As will be discussed in Section 4, the effect of phase space diffusion appears as a pinch flux in the real (coordinate) space.

It is worth noting that within the low $\beta$ approximation, we should not. distinguish between the curvature and gradient tems in the magnetic drift velocity [14]. Along with the full curvature effect we wonld have to include the parallel magnetic drift [14] as well as some other terms in Eys. (11) and (12) to satisfy Eq. (5). (Guiding center equations for a finite s can be found in [8].) 


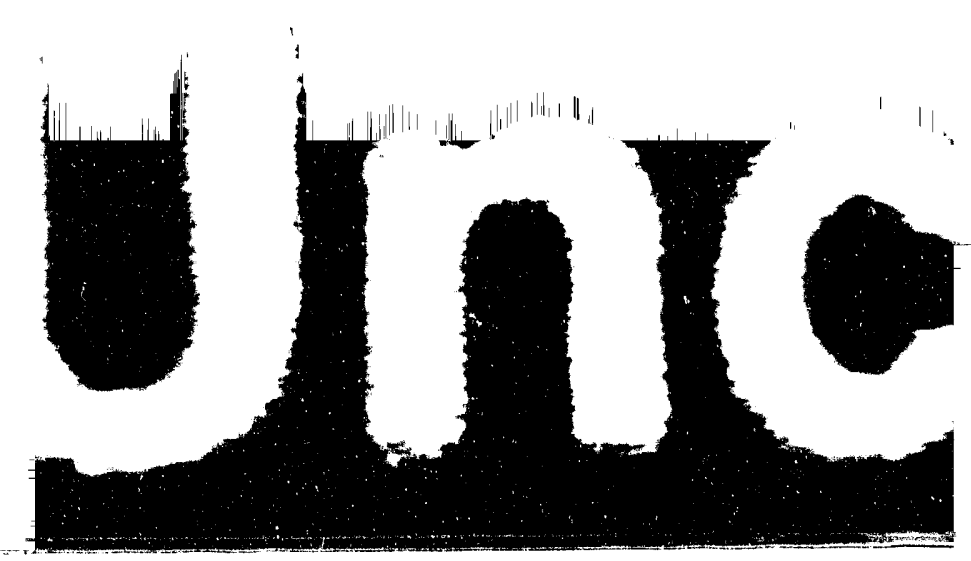

\section{$3 \quad$ Reduced Transport Equations}

In this Section we derive the reduced transport equations in the drift approximation. These equations are relevant for a plasma with low frequency $\left(\omega<\omega_{B_{1}}\right)$, long wavelength fluctuations $\left(k_{\perp} \rho_{i}<1\right)$. Averaging Eq. (1) with proper weights and using Eqs. (10)-(12) we arrive at the following moments equations:

$$
\begin{gathered}
\frac{\partial n}{\partial t}+\nabla \cdot\left(n V_{\|} \mathbf{h}+n \mathbf{V}_{E}+\frac{1}{m \omega_{B}} \mathbf{h} \times \nabla \ln B\left(2 p+\pi_{\|} / 3\right)\right)=0 \\
m n\left(\frac{\partial}{\partial t}+\mathbf{V}_{E} \cdot \nabla\right) V_{\|}=e n \mathbf{E} \cdot \mathbf{h}+m n V_{\|} \mathbf{V}_{E} \cdot \nabla \ln B-\mathbf{h} \cdot \nabla p \\
-\frac{2}{3} \mathbf{h} \cdot \nabla \pi_{\|}-\pi_{\|} \nabla \cdot \mathbf{h}, \\
+\nabla \cdot\left(\mathbf{V}_{E} \frac{m n V_{\|}^{2}}{2}+\frac{3}{2} p \mathbf{V}_{E}+\frac{T}{m \omega_{B}} \mathbf{h} \times \nabla \ln B\left(i j p(1+1)+7 \pi_{\|} / 6-\pi_{\|}^{*} / 3\right)\right) \\
=e n V_{\|} \mathbf{E} \cdot \mathbf{h}+\frac{q}{m \omega_{B}} \mathbf{h} \times \nabla \ln B \cdot \mathbf{E}\left(2 p+\pi_{\|} / 3\right)
\end{gathered}
$$

Here $\pi_{\|}, \pi_{\|}^{*}$ and $\chi$ are given by the relations

$$
\begin{gathered}
\pi_{\|}=m \int\left(v_{\|}^{2}-\frac{v_{1}^{2}}{2}\right) L_{0}^{(5 / 2)}(x) f d^{3} u, \quad \pi_{\|}^{*}=m \int\left(v^{2} \|^{2}-\frac{u_{1}^{2}}{2}\right) L_{1}^{(5 / 2)}\left(. v^{\prime}\right) f d^{3} u^{\prime}, \\
\gamma=\frac{8}{15 n} \int L_{2}^{(1 / 2)}(x) f d^{3} v,
\end{gathered}
$$

where $L_{n}^{(\alpha)}$ are the generalized Laguerre polynomials with the argument $x=$ $v^{2} / v_{t}^{2}$. It can be shown that the same equations may be obtained also from the standard Braginskii fluid equations [15] supplemented by the (irad-type equation for the heat flux by an expansion in $1 / B$ as a the small paraneter [16]. The drift transport equations of a similar strusture have been obtained in $[12,16]$.

Eqs. (13)-(15) contain the effects of transport associated with fluctuations as well as standard neorlassical transport. It ran be shown that the 
standard neoclassical fluxes are represented by the last term in parentheses in Eq. (13) (density flux) and the last term in second parentheses in Eq. (15) (heat flux). The last term on the r.h.s. of Eq. (15) describes the neoclassical energy transfer [17]. This term also determines the specific anomalous energy exchange due to fluctuations. The fluid moments $n, T, p$, etc. in Eqs. (13)-(15) contain both equilibrium and fluctuating parts including effects associated with toroidal particle drift. They may also include the resonant effects of perturbations due to the kinetic effects [18]. It is noted that the convective energy flux associated with electrostatic fluctuations is given by the term $\frac{3}{2} \mathbf{V}_{E} p$ (due to cancellation of the diamagnetic convective part and cross-heat flux $[5,19])$, while the the anomalous energy flux due to magnetic fluctuations is determined by the contributions from the free parallel streaming and pressure anisotropy (the terms $\frac{5}{2} p V_{\|} \mathbf{h}$ and $\frac{2}{3} \pi_{\|} V_{\| h} \mathrm{~h}$ in Eq. (15) respectively). From this one can conclude that in general the convective energy fluxes due to electrostatic and magnetic fluctuations are asymmetric [20]. However if we consider only the resonant part of the magnetic transport associated with particles with small parallel velocities, ${ }^{\prime \prime} \| \ll l^{\prime} \perp$, then we observe that the convective transport due to magnetic fluctuations reduces to the form $\frac{5}{2} p V_{\|} \mathrm{h}+\frac{2}{3} \pi_{\|} V_{\|} \mathrm{h} \simeq \frac{3}{2} p V_{\|} \mathrm{h}$, so that the convertive fluxes due to magnetic and electrostatic fluctuations becomes symmetric with the same numerical factor $3 / 2$.

The perturbation of plasma density, temperature, etc. related to electrostatic potential fluctuations may also contain the poloidal variation due to toroidicity. The effect of poloidal variations of the espuilibrium electric field in the neoclassical theory have been considered in [2], 22]. The similar effect for fluctuations yields the pinch terms in the transport fluxes. It has beetn shown that these types of effects are related to the gradients of the magnetic field. We discuss this further in the next Section.

\section{Specific Anomalous Transport Due To Com- bined Effects Of Fluctuations And Gradi- ent Of Magnetic Field}

In this Section we present a simple estimate of the transport flux related to the gradient of a magnetic field. In terms of kinetic energy $\varepsilon$ and addabatic 
moment $\mu$, the drift-kinetic equation has the form $[2: 3,24]$

$$
\begin{gathered}
\frac{\partial f}{\partial t}+v_{\|} \mathbf{h} \cdot \nabla f+\mathbf{V}_{E} \cdot \nabla f+\mathbf{V}_{d} \cdot \nabla j+\frac{q}{m} \mathbf{E} \cdot \mathbf{h} \frac{\partial f}{\partial v^{\prime} \|} \\
+\left(v_{\perp}^{2} / 2+v_{\|}^{2}\right) \mathbf{V}_{E} \cdot \nabla \ln B \frac{\partial f}{\partial \mathcal{E}}=0 .
\end{gathered}
$$

The last term on the r.h.s of this equation is responsible for the diffusion in velocity space caused by fluctuations. Though this term is well known in neoclassical transport theory [23] and in the theory of linear instabilities in a toroicial plasma $[24,25]$, it has not been incorporated in the problem of anomalous transport. In particular, it has not been taken into account. in calculations of anomalous fluxes in toroidal geometry $[26,27]$. This term yields the anomalous energy exchange effect given by the last term on r.h.s of Eq. (15), the effects of which cannot be recovered in the slab plasma approximation. Another consequence is an additional contribution to the perturbed distribution function which is proportional to the gradient of the magnetic field and fluctuations, so that, in general, the transport fluxes in a toroidal plasma cannot be cast into a sum of the neoclassical and slabtype anomalous fluxes [27]. From Eq. (16) we find the perturbation of the distribution function in the form

$$
\hat{f}=-i G_{k \omega}\left(\widetilde{\mathbf{V}}_{E} \cdot \frac{\nabla f_{0}}{f_{0}}-\frac{q}{T} v_{\|} \tilde{\mathbf{E}} \cdot \mathbf{h}-\frac{v_{\perp}^{2}+2 v_{\|}^{2}}{l_{t}^{2}} \widetilde{\mathbf{V}}_{E} \cdot \frac{\nabla B}{B}\right) f_{0},
$$

where $G_{k \omega}^{-1}=\left(\omega-k_{\|} v_{\|}-\omega_{d}+\Delta \omega_{N L}\right)$ is the particle propagator (which may also include the effects of nonlinear broadening and frepuency shift.) and $\omega_{d}=\left(v_{\perp}^{2} / 2+v_{\|}^{2}\right) \mathbf{k} \cdot \mathbf{h} \times \nabla \ln B / \omega_{B}$ is the magnetic drift frecfuency. One can see that the perturbed distribution function contains the part proportional to the gradient of the equilibrium magnetic field (last term on the r.h.s. of Eq. (17)) in addition to the standard term proportional to the gradients of the equilibrium plasma temperature and density. (Other terms in Eq. (17) leading to neoclassical transport have been omitted.) From the comparison of this term with the first term on the r.h.s of Eq. (17) one can see that the gradient of a magnetic field can drive an anomalous Hux in the same way as the gradient of the equilibrium distribution function (due to gradients of density and temperature) does. This additional term, which is proportional to the gradient of the equilibrium magnetic field. gives rise to a pinch effect 
in the real space and describes diffusion in the phase space. It can be readily seen that the pinch is directed toward the region of higher magnetic field. The net flux is the result of an in-out difference in the surface elements due to the toroidicity, $d S=d S_{0}(1+\epsilon \cos \theta)$, (for the magnetic field $\mathbf{B}=B_{0}\left(\hat{e}_{\zeta}+\right.$ $\left.\Theta \hat{e}_{\theta}\right)(1-\epsilon \cos \theta)$, where $\left.\Theta=\epsilon / q, \epsilon=r / R\right)$. The perturbations of plasma parameters such as density, pressure, etc. associated with the discussed term are proportional to the electrostatic field and also have poloidal dependence. Physically, the poloidal variations of perturbed plasma parameters arise due to the particle toroidal drift off the magnetic surface and they typically scale as a ratio of the banana width to the wavelength of the fluctuations. The sorresponding energy flux is given by

$$
Q_{p}=\frac{3}{2}\left\langle\mathbf{V}_{E} \tilde{p}^{\theta}(1+\epsilon \cos \theta)\right\rangle
$$

For the simplest case of a toroidal propagator in the form $\left(i_{k w}=i \pi \delta\left(v_{\|} / q R\right)\right.$ (plateau regime) the perturbation of ion pressure may be estimated as $\tilde{p}^{\theta} / p_{0} \simeq$ $q k_{\perp} \rho_{i} e \Phi / T\left(\tilde{p}^{\theta}=\tilde{p}^{c} \cos \theta+\tilde{p}^{s} \sin \theta\right)$. This gives a pinch velocity of the gyroBohm type with an additional factor $\epsilon q$ (only the part proportional to $\nabla \ln B$ is retained)

$$
V_{0}=\epsilon q \frac{c T}{e B} \rho_{i} / L_{n}^{2}
$$

Effect of the same order appears as an anomalous energy exchange (see the last term in Eq. (15)). These effects are intrinsically related to the PfirschShlüter current effects in a toroidal plasma with fluctuations discussed in [28].

For the banana regime we will obtain a banand reduced Bolnn scaling instead of Eq. (19). In the strong turbulence case with a nonlinear propagator of the order of the drift frequency we obtain the Bohm-type scaling for the pinch velocity. Generally, the same effect arises also in the density flux; however it must be considered together with the ambipolarity constraint. imposed by the quasineutrality condition [21].

For the quasilinear case the magnetic gradient term can be formally removed from the expression in (17) by using the linear resonant condition $\omega=k_{\|} v_{\|}+\omega_{d}$, so that the perturbed distribution function becomes proportional to the difference between the drift frequency and the eigenfrequency $\hat{f} \sim\left(\hat{\omega}_{*}-\omega\right)$, where $\hat{\omega}_{*}=c T \mathbf{k} \cdot \nabla f_{0} / \epsilon B f_{0}[24,25]$. However in the lat ter case 
we must take into account the toroidal contribution to the eigenfrequency [5]. so the magnetic drift term again reappears in the perturbed distribution function. The effect of the magnetic drift in the transport flux is typically less than that of the slab $\mathbf{E} \times \mathbf{B}$ drift by a factor $\epsilon L_{n} / L_{B}$ (however, the additional small parameter $\epsilon$ appearing due to surface averaging may be removed if we assume a strong ballooning mode structure). Note that this effect increases towards the plasma periphery (it might be especially important in the vicinity of the outer separatrix in divertor configurations).

\section{Conclusion}

Reduced transport equations for the toroidal plasma with fluctuations have been derived. These equations include both the anomalous transport effects and the standard neoclassical transport. The structure of convective energy fluxes in a plasma with electrostatic and magnetic fluctuations have been considered. The effect of a magnetic field inhomogeneity on the anomalous transport was investigated. The magnetic field inhomogeneity is identified as an additional source of an anomalous transport. It is shown that the combined effect of fluctuations and a gradient of the equilibrium magnetic field naturally produce pinch type transport fluxes.

\section{Acknowledgements}

One of the authors (A.S) is indebted to P. Yushmanov for useful discussions.

This work was supported by the Natural Sciences and Engineering Research Council of Canada and U.S. DOE grant No DE-F(i)2-S(GER53218.

\section{References}

[1] Callen J.D., Christiansen J.P., Cordey J.D., Thomas P.R. and Thomsen K.. Nuclear Fusion 27, 1857 (1987).

[2] Dnestrovskii Yu.N., Neudachin S.V. and Pereverzev (i.V.. Sus. J. Plasma Phys. 10, 137 (1984).

[3] Terry P.W., Phys. Fluids B 1, 1932 (1989).

[4] Itoh Sanae-I., Phys. Fluids B 4, 796 (1992). 
[5] Nordman H. and Weiland J., Nuclear Fusion 29, 251 (1989).

[6] Lichtenberg A and Liberman M., Regular and Stochastic Motion, Springer, 1983.

[7] Mynik H.E. and Duval R.E., Phys. Fluids B1, 750 (1989).

[8] Sivukhin D.V., in Reviews of Plasma Physics, edited by M.A. Leontovich (Consultants Bureau, New York), vol.1, p.1 (1965).

[9] Hazeltine R.D. and Ware A.A., Plasma Phys. 20. 673 (1978).

[10] Morozov A.I and Solov'yev L.S., in Revieus of Plasma Physics, edited by M.A. Leontovich (Consultants Bureau, New York), vol.2. p.201 (1966).

[11] Kadomtsev B.B., in Plasma Physics and the Problem of Controlled Thermonuclear Reactions, ( Pergamon Press, London), Vol. 4, p.438 (1959).

[12] Mikhailovskii A.B. and Tsypin V.S., Sov. Phys. JETP 56, 75 (1982).

[13] Weiland J., Phys. Fluids B 4, 1388 (1992).

[14] Hazeltine R.D. and Meiss J.D. Plasma C'onfinement (Addison-Wesley), p.122 (1992).

[15] Braginskii S.I., in Reviews of Plasma Physics, edited by M.A. Leontovich (Consultants Bureau, New York), Vol.1, p.205 (1965).

[16] Mikhailovskii A.B. and Tsypin V.S., Plasma Physics 13, 785 (1971); Mikhailovskii A.B. Electromagnetic Instabilitics in an Inhomogeneous Plasma, Institute of Physics Publishing, Bristol, p.147 (1992).

[17] Ware A.A., Nuclear Fusion 21, 631 (1981).

[18] Chang Z. and Callen J.D., Phys. Fluids B4, 1167 (1992): Wang J.P. and Callen J.D., Phys. Fluids B4, 1139 (1992).

[19] Tsai S., Perkins F.W. and Stix T.H., Phys. Fluids 13, $210 \times(1970)$.

[20] Smolyakov A.I. and Hirose A., Comments Plasma Phys. (ontr. Fusion 15. 281 (1993).

[21] Stringer T.E. Phys. Fluids B 3, 981 (1991). 
[22] Hazeltine R.D. and Ware A.A., Phys. Fluids 19, 11633 (1976).

[23] Frieman E.A., Phys. Fluids 13, 490 (1970).

[24] Mikhailovskii A.B., Nuclear Fusion 13, 259 (1973).

[25] Catto P.J. and Rosenbluth M.N., Phys. Fluids 24, 243 (1981).

[26] Shaing K.C., Phys. Fluids 31, 2249 (1988).

[27] Balescu R., Phys. Fluids B 2, 2100 (1990).

[28] Wobig H., in 18th EPS Conference Contr. Fusion Plasma Phys. (Berlin, 1991), vol. 15C, Pt.IV, p.213. 

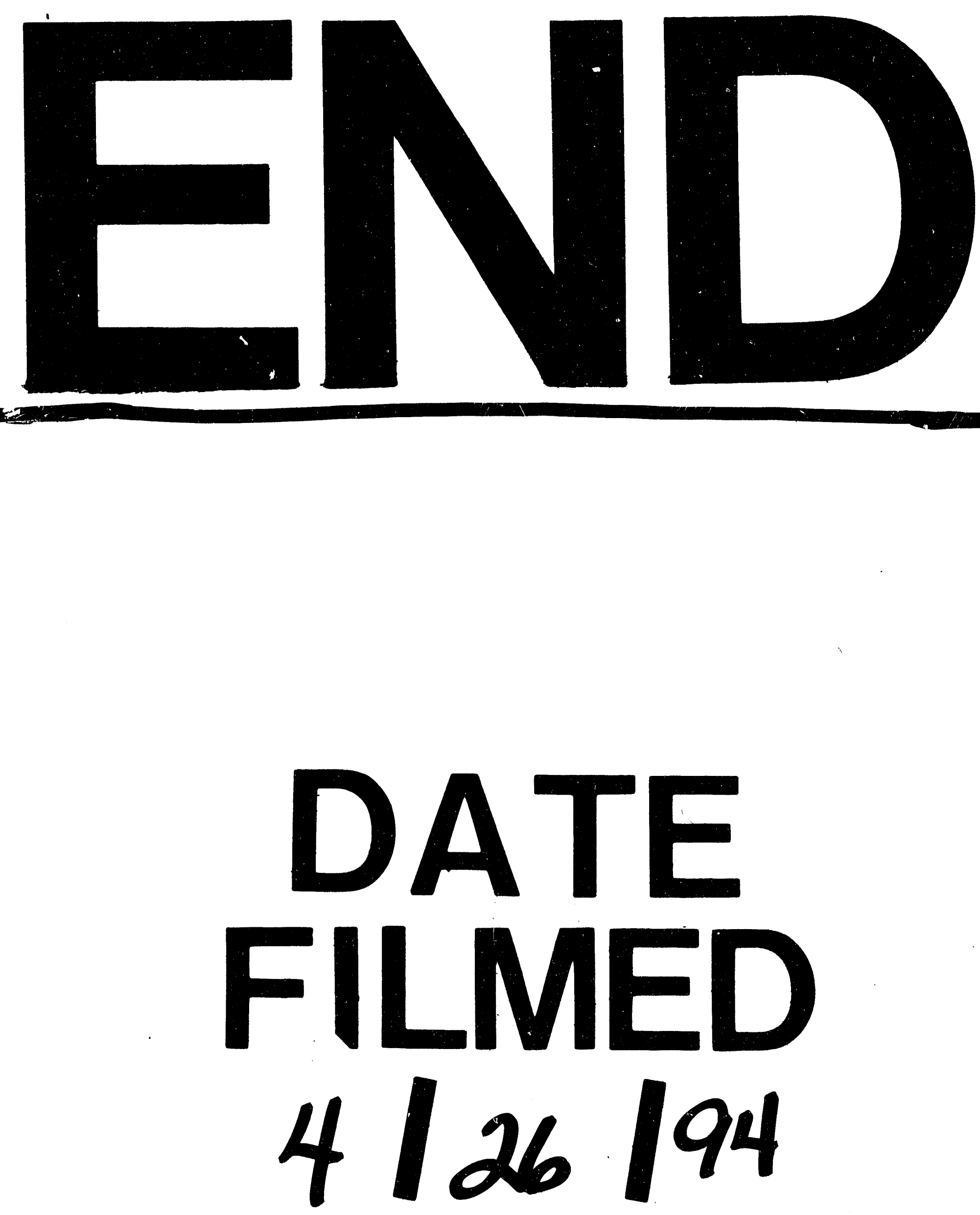


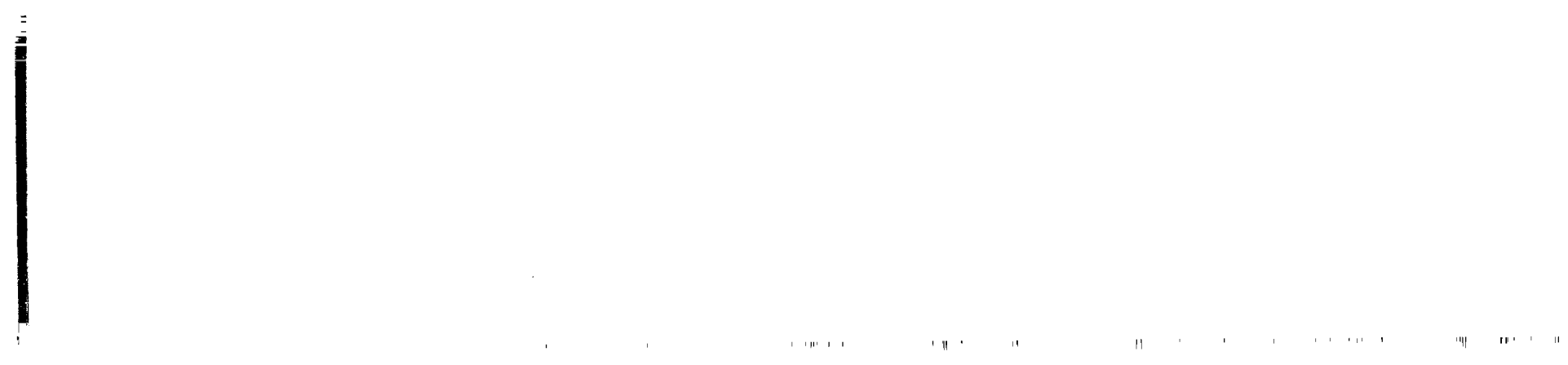

